\title{
MediterrAsian Diet Products That Could Raise HDL-Cholesterol: A Systematic Review
}

\author{
Mariangela Rondanelli, ${ }^{1}$ Attilio Giacosa, ${ }^{2}$ Paolo Morazzoni, ${ }^{3}$ Davide Guido, ${ }^{4}$ \\ Mario Grassi, ${ }^{5}$ Gabriella Morandi, ${ }^{5}$ Chiara Bologna, ${ }^{1}$ Antonella Riva, ${ }^{3}$ \\ Pietro Allegrini, ${ }^{3}$ and Simone Perna ${ }^{1}$ \\ ${ }^{1}$ Department of Public Health, Experimental and Forensic Medicine, School of Medicine, Endocrinology and Nutrition Unit, \\ University of Pavia, Azienda di Servizi alla Persona di Pavia, Pavia, Italy \\ ${ }^{2}$ Department of Gastroenterology, Policlinico di Monza, 20900 Milan, Italy \\ ${ }^{3}$ Research and Development Department, Indena SpA, 20139 Milan, Italy \\ ${ }^{4}$ Department of Public Health Experimental and Forensic Medicine, Unit of Biostatistics and Clinical Epidemiology, \\ University of Pavia, Pavia, Italy \\ ${ }^{5}$ Department of Brain and Behavioral Sciences, Section of Biostatistics, Neurophysiology and Psychiatry, University of Pavia, \\ Pavia, Italy
}

Correspondence should be addressed to Simone Perna; simoneperna@hotmail.it

Received 16 June 2016; Accepted 16 August 2016

Academic Editor: Giuseppe Caminiti

Copyright (C) 2016 Mariangela Rondanelli et al. This is an open access article distributed under the Creative Commons Attribution License, which permits unrestricted use, distribution, and reproduction in any medium, provided the original work is properly cited.

Background. High HDL-cholesterol (HDL-C) values are negatively correlated with cardiovascular diseases. This review analyses the effect of the supplementation with various Mediterranean diet products (artichoke, bergamot, and olive oil) and Asian diet products (red yeast rice) on the HDL-C value in dyslipidemic subjects. Methods. A systematic review has been done involving all the English written studies published from the 1st of January 1958 to the 31st of March 2016. Results. The results of this systematic review indicate that the dietary supplementation with red yeast rice, bergamot, artichoke, and virgin olive oil has promising effects on the increase of HDL-C serum levels. The artichoke leaf extract and virgin olive oil appear to be particularly interesting, while bergamot extract needs further research and the effect of red yeast rice seems to be limited to patients with previous myocardial infarction. Conclusions. Various MediterrAsian diet products or natural extracts may represent a potential intervention treatment to raise HDL-C in dyslipidemic subjects.

\section{Introduction}

Cardiovascular disease (CVD) remains the leading cause of morbidity and mortality worldwide [1]. Hyperlipidemia, resulting from the abnormalities of lipid homeostasis, is a common risk factor for the development of CVD [2]. It is well known that the increase of total cholesterol and particularly of LDL-cholesterol (LDL-C) is positively associated with the risk of CVD, while high values of HDL-cholesterol (HDL-C) are inversely correlated with the risk of CVD [3]. A metaanalysis of four prospective studies (the Framingham Heart Study, the Multiple Risk Factor Intervention Trial (MRFIT), the Lipid Research Clinics Prevalence Mortality Follow-Up Study, and the Coronary Primary Prevention Trial) showed that every $1 \mathrm{mg} / \mathrm{dL}^{-1}\left(0.026 \mathrm{mmol} / \mathrm{L}^{-1}\right)$ increase in HDL-C is associated with a significant coronary heart disease (CHD) risk reduction of $2 \%$ in men and $3 \%$ in women [4].

From the pharmacological point of view, statins represent the treatment of choice to reduce the serum levels of total and LDL-cholesterol. However, in both primary and secondary prevention trials with statins, only small, insignificant increases in HDL-cholesterol were achieved. In the Air Force/Texas Coronary Atherosclerosis Prevention Study (AFCAPS/TexCAPS), treatment with lovastatin 
resulted in a 6\% mean increase in HDL-cholesterol [5]; in the West Of Scotland Coronary Prevention Study (WOSCOPS), treatment with pravastatin resulted in a 5\% increase [6]; in the Cholesterol and Recurrent Events (CARE) trial, pravastatin also increased HDL-cholesterol by 5\% [7]; and in the Scandinavian Simvastatin Survival Study (4S), simvastatin increased HDL-cholesterol by $8 \%$ (insignificant when corrected for the placebo group) [8].

Therefore, at the present time, there is no clear indication that HDL-cholesterol elevation by statins translates into clinical benefit [9].

Interesting effects on the serum lipid profile are associated with behavioral interventions. Healthy diet and physical exercise have beneficial effects on the improvement of the serum lipid profile, with reduction of total cholesterol (TC), triglycerides (TG), and LDL-C with increase of HDL-C [10]. A healthy diet may include reducing the intake of saturated and trans fatty acids and dietary cholesterol and increasing the intake of fish, fruit, and vegetables. Typically, this objective is achieved with Mediterranean diet, rich in olive oil, fruits and vegetables, whole cereals, nuts, legumes, fish, and red wine [11] and with red yeast rice which is a typical product of the Asian diet [12]. The combined preventive role of these two diet models has been defined as "MediterrAsian diet" [13]. Recent data demonstrate that various MediterrAsian products and botanical extracts such as the extracts of artichoke, bergamot, red yeast rice, or monacolin and olive oil may increase HDL-cholesterol.

Other food or food derived components could be efficacious in favorably modulating human serum HDL-C, such as ethanol and eggs. Prior and recent evidence shows favorable changes in HDL-C, other CVD risk factors, and CVD event rates with moderate and regular ethanol intake [14]. However, the application of these findings in clinical practice remains problematic, due to the lack of randomized, controlled clinical trials and due to the potential hazards of ethanol consumption. As far as eggs are concerned, multiple research data and even meta-analyses have been conducted to investigate the effects of eggs on serum cholesterol level and cardiovascular health with different conclusions [15]. Convincing data have been obtained on the increase of HDL$\mathrm{C}$ after egg consumption in patients with metabolic syndrome [16], but not in healthy subjects.

The purpose of this systematic review is to investigate the effect of the supplementation with the four typical MediterrAsian products previously described (extracts of artichoke and bergamot, red yeast rice or monacolin, and olive oil) on high-density lipoprotein cholesterol (HDL-C).

\section{Methods}

The present systematic review was performed according to the following steps suggested by Egger et al. [17]: (i) configuration of a working group; (ii) formulation of the revision question on the basis of considerations made in the abstract; (iii) identification of relevant studies. The search involved all the studies published from the 1st of January 1958 to the 31st of March 2016. English written articles were identified by searching the Medline database [18], Scopus
[19], ISI Web of Science [20], and Google Scholar [21]. The analysis was carried out in the form of a systematic review of the reports.

2.1. Inclusion and Exclusion Criteria. Two reviewers (Simone Perna and Mariangela Rondanelli) independently reviewed each report. For each of the relevant abstracts, full publications were retrieved for evaluation on the basis of criteria established a priori. Original clinical trials investigating the effects of botanical extract supplementations as red yeast rice, monacolin $\mathrm{k}$, bergamot, artichoke, and olive oil and their eventual comparison with a control diet (if available) were evaluated. The change of high-density lipoprotein cholesterol (HDL-C) was the primary outcome and no secondary outcomes were considered.

All adult age groups were included. The eligible studies were required to report baseline and follow-up values, that is, the mean change from baseline ( $\Delta$-change) and/or the mean difference $\Delta$-changes (MD $\Delta$ ) between intervention groups for HDL-C outcome. Trials were included also if a botanical extract supplementation was associated with the supplementation of other foods. Figure 1 reports the flow diagram of the study divided by botanical supplementation.

2.2. Data Collection. The following data were extrapolated from all the revised studies: (i) author and year of publication; (ii) number of participants for each study; (iii) mean age of the subjects; (iv) inclusion criteria; (v) dietary supplement (in intervention and control groups); (vi) duration of intervention (in weeks); (vii) mean baseline HDL-C; (viii) mean postintervention HDL-C; (ix) mean change from baseline ( $\Delta$-change); (x) mean difference $\Delta$-change $(\mathrm{MD} \Delta)$ between intervention groups for HDL-C outcome ( $P$ values); (xi) study design and level of evidence (as suggested by Centre for Evidence-Based Medicine [29]).

The obtained data were summarized in four tables (Tables 1-4). Each table shows study design, level of evidence, inclusion criteria, sample size, baseline mean age, dietary intervention $\backslash$ control, and duration. HDL-C related measurements, that is, $\Delta$-changes and/or MD $\Delta$, if available, were included. In order to define the type of intervention, we focused on the dosage of supplementation (grams per day), on the number of supplementation days, on the number of patients (control and $\backslash$ or intervention group), and on the type of control diet.

\section{Results}

3.1. Red Yeast Rice and Monacolin K. Concerning the effects of red yeast rice on HDL-C, the literature search based on the keywords ["Monacolin k" OR "Monascus Purpureus" OR "Red Yeast Rice"] AND ["HDL" OR "cholesterol" OR "high density lipoproteins"] retrieved 82 articles. After screening, 69 papers were selected for full-text revision. After applying our inclusion and exclusion criteria, 62 studies were excluded and 7 clinical trials were selected for the present systematic review. Figure 1 shows the study selection process. The 7 clinical trials included a total of 5444 adults (4224 females, 1620 males). 

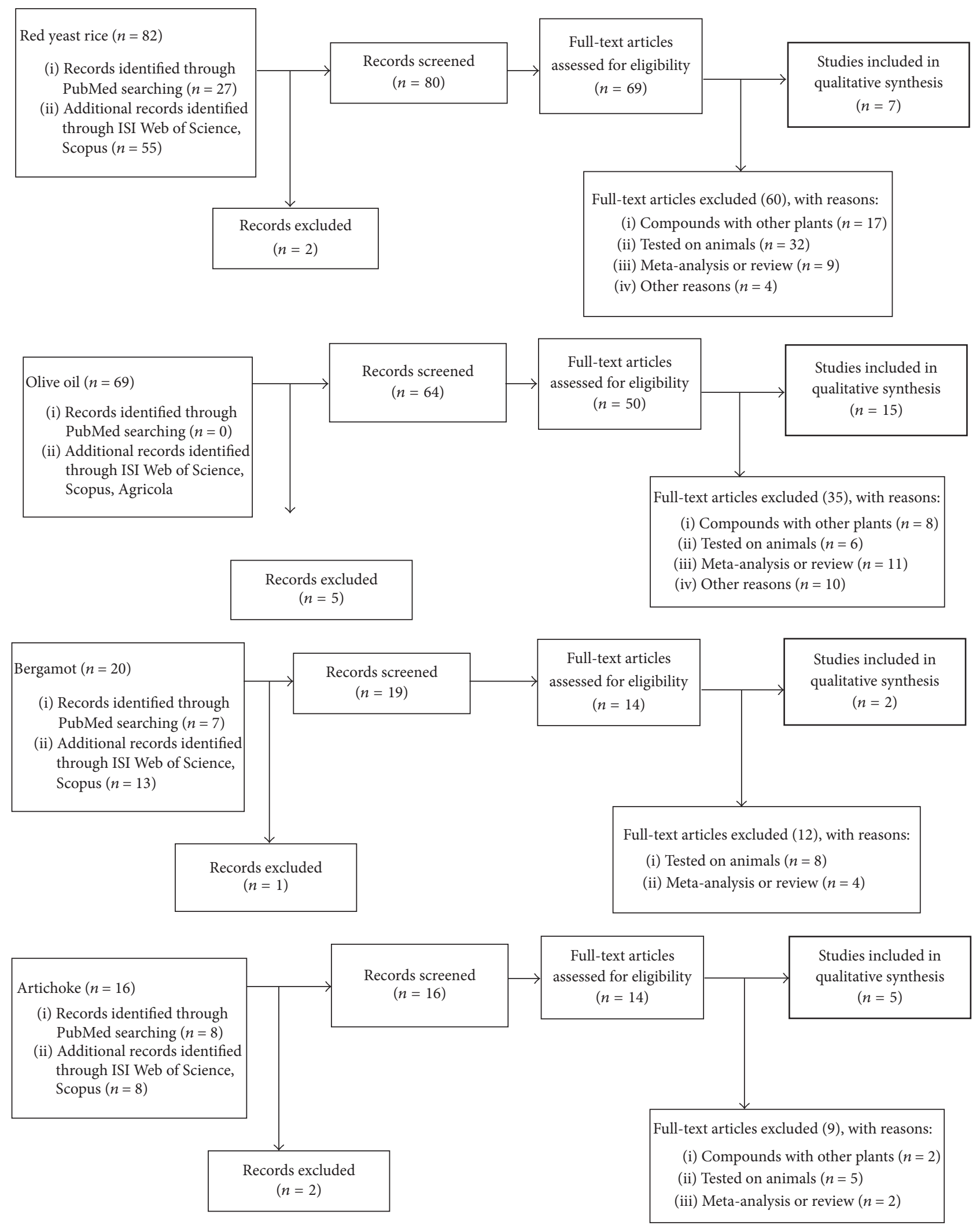

FIGURE 1: Flow diagram of the study divided by botanical supplementation. 


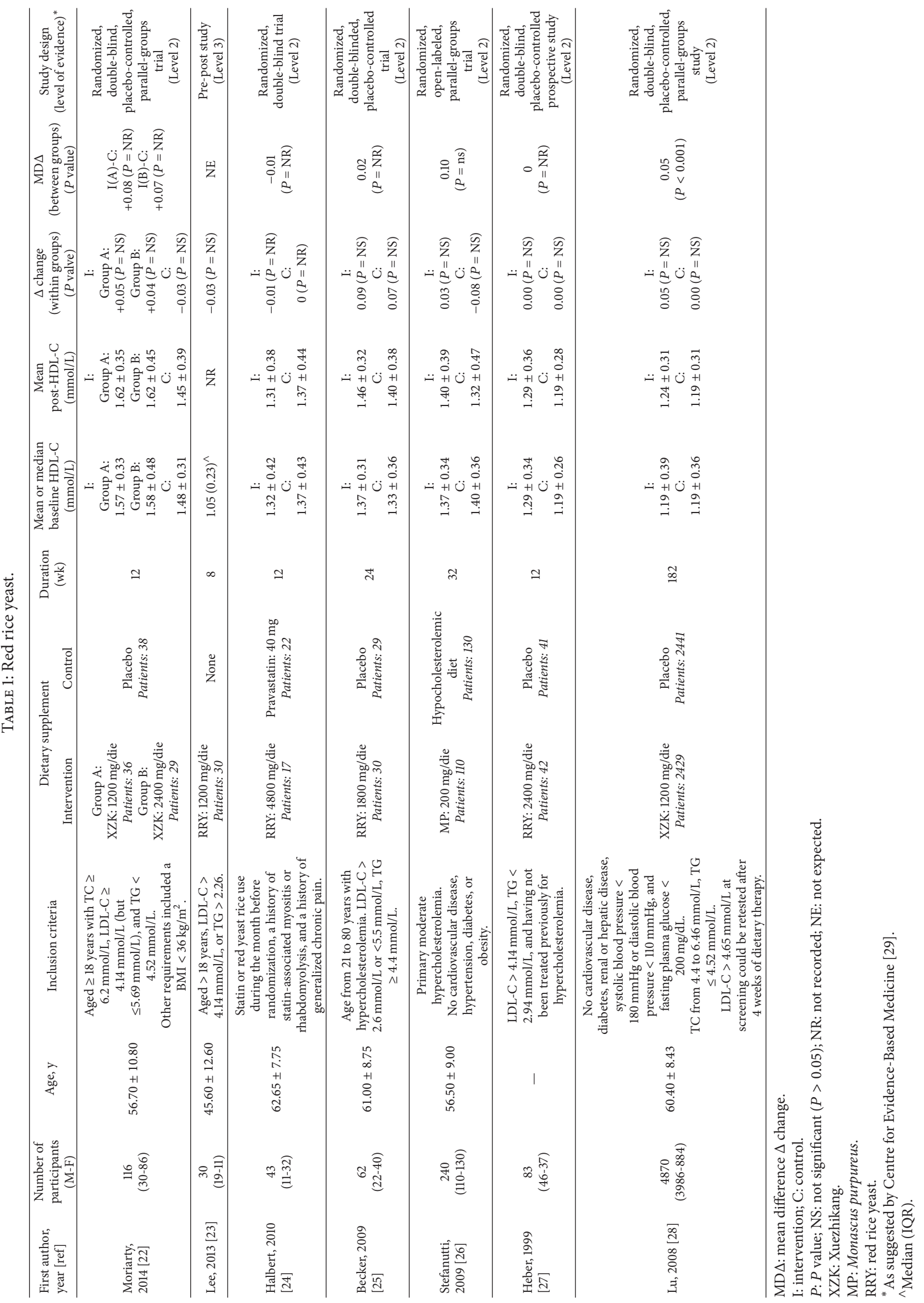




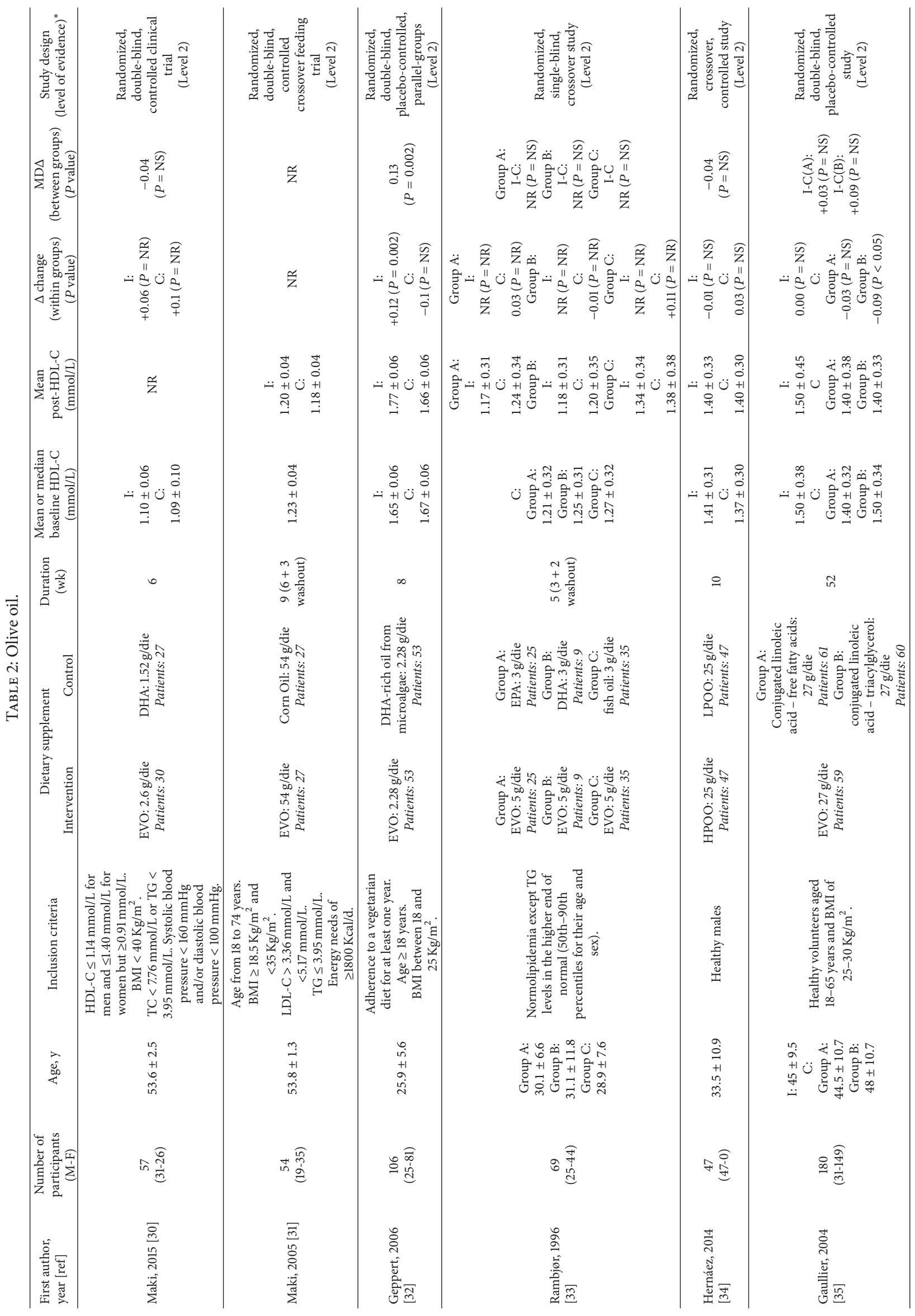




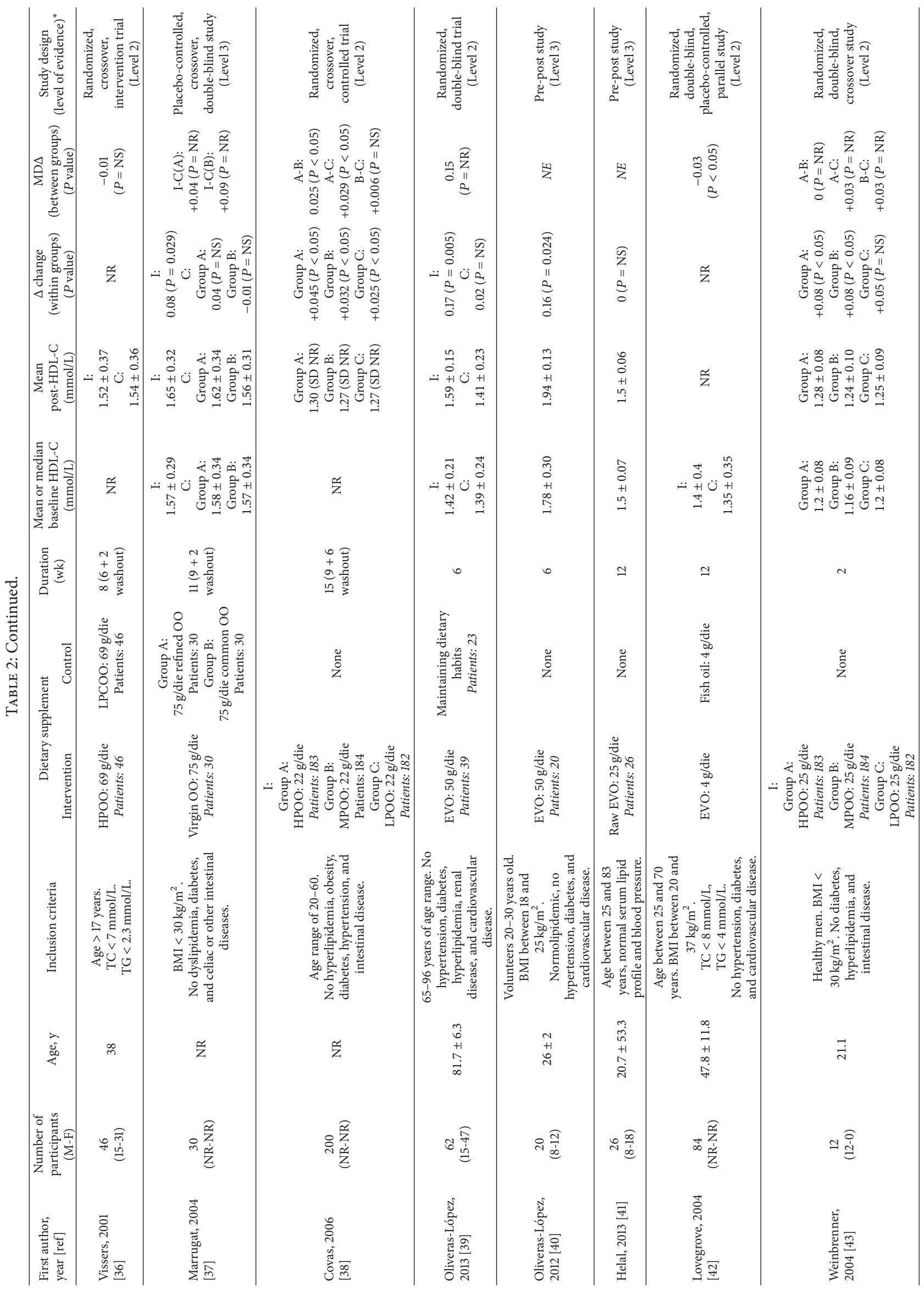




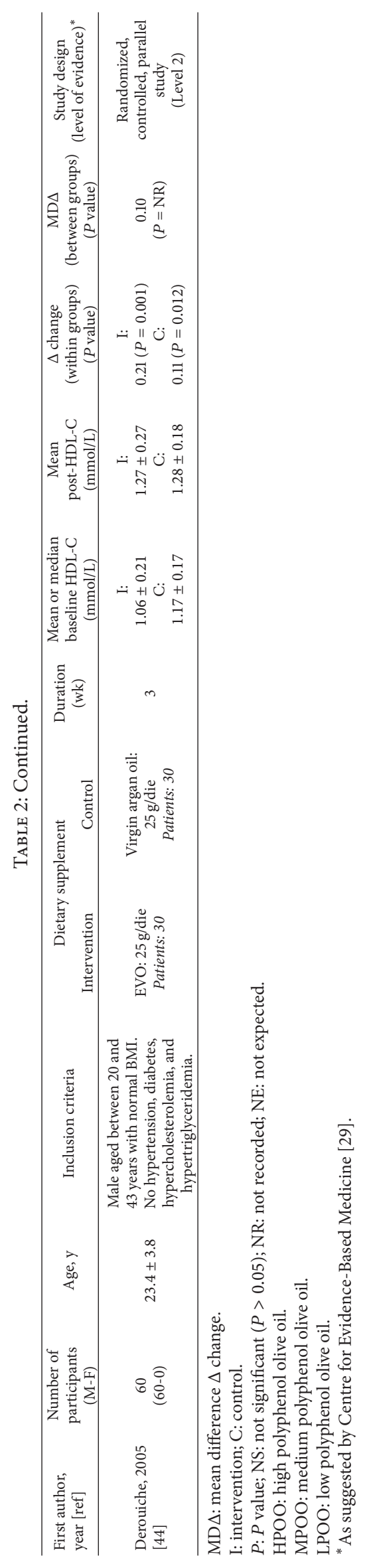



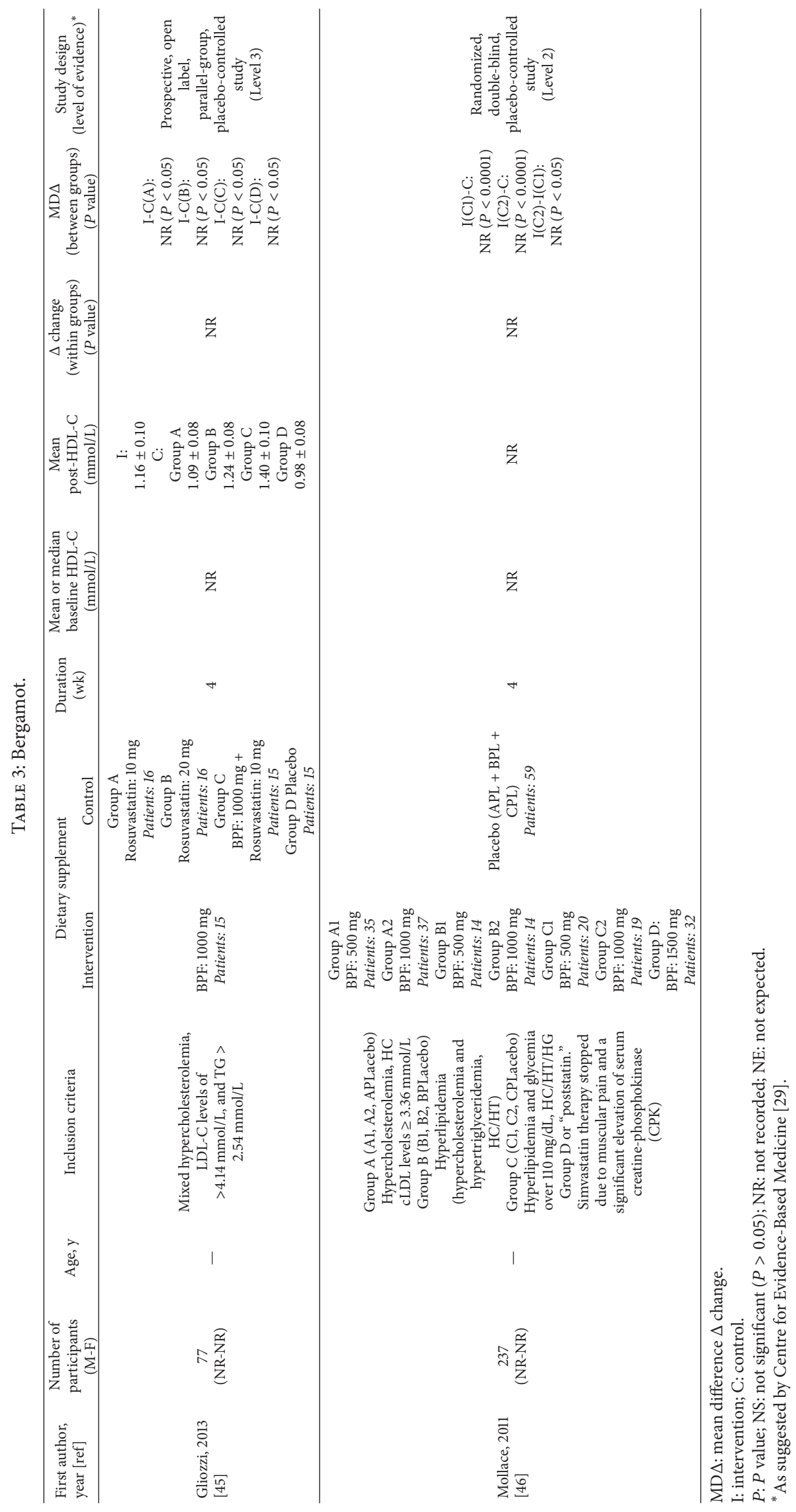


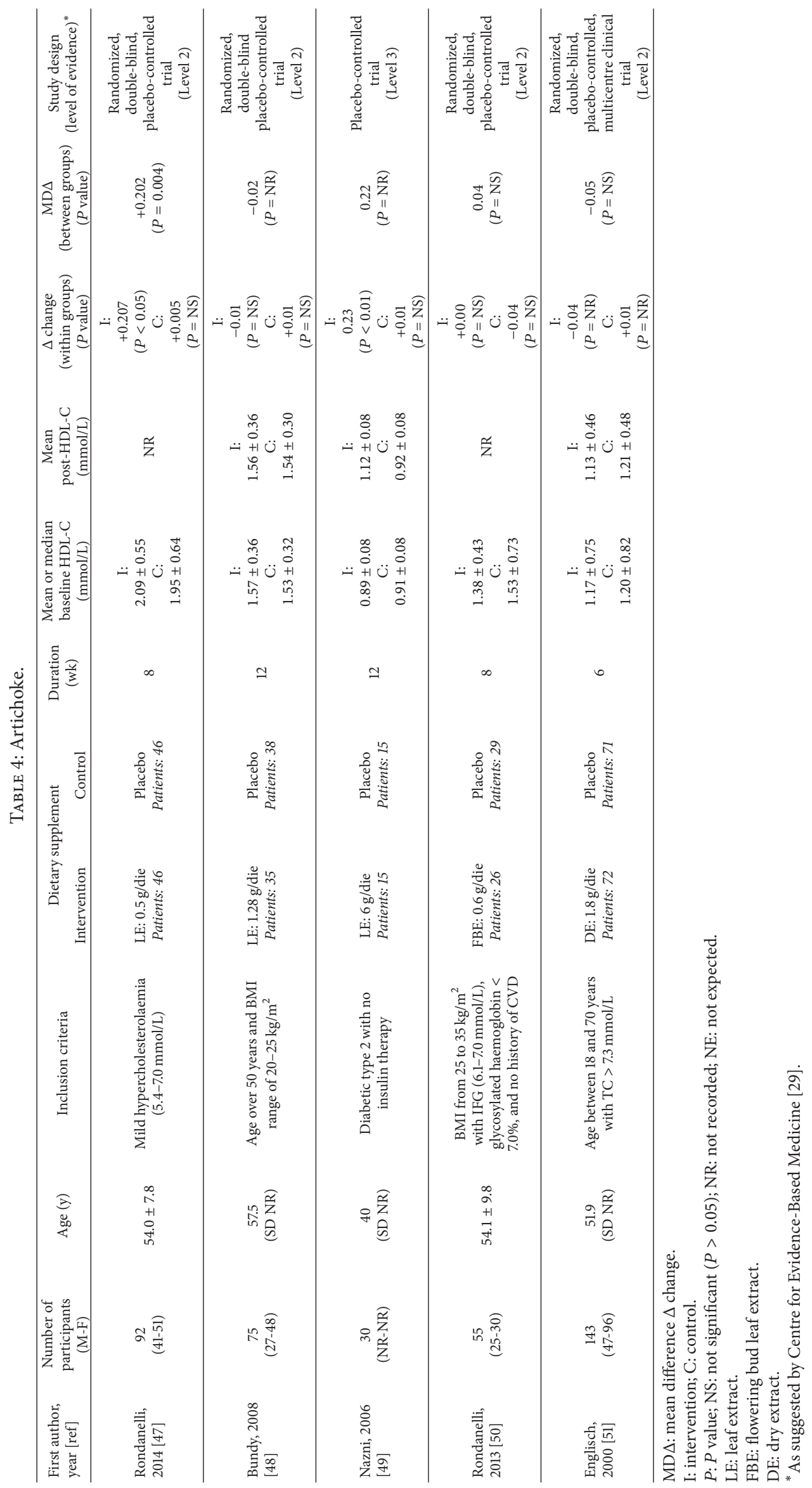


The trial characteristics are outlined in Table 1. Regarding the study design, out of the 7 selected studies, two were randomized, double-blind, placebo-controlled, parallelgroups trials $[22,28]$, two were randomized, double-blind, and placebo-controlled trials [25, 27], one was a randomized and double-blind trial [24], one was a randomized and open label parallel trial [26], and one was a pre-post study [23].

Concerning the control group, four studies $[22,25,27$, 28] were placebo-controlled, one study [24] was performed versus pravastatin $40 \mathrm{mg} / \mathrm{day}$, and one [26] was performed versus a hypocholesterolemic diet. The study of Lee et al. [23] had no control group.

The baseline characteristics and disease status of the participants varied. Four studies [22, 23, 25, 27] included subjects with hypercholesterolemia; two studies [26, 28] included subjects with moderate hypercholesterolemia or normal lipid levels. The remaining study [24] included subjects (i) with statin or red yeast rice treatments during the month before randomization, (ii) with a history of statinassociated myositis or rhabdomyolysis, and (iii) with a history of generalized chronic pain.

Dietary interventions lasted from 8 to 216 weeks, with a dosage of red rice yeast ranging from 1200 to $4800 \mathrm{mg} / \mathrm{die}$ or a dosage of Xuezhikang (XZK) ranging from 1200 to $2400 \mathrm{mg} / \mathrm{die}$ or a dosage of Monascus purpureus of $200 \mathrm{mg} / \mathrm{die}$.

The study by $\mathrm{Lu}$ et al. [28] based on a large number of cases (4870) showed a statistically significant increase of HDL-C $(\Delta \%=+4.2 \%, P<0.001)$ after supplementation with XZK versus placebo, while the other six studies did not show significant changes of HDL-C. The randomized, placebocontrolled, multicentre study coordinated by Lu et al. was conducted to evaluate the effects of XZK, a partially purified extract of red yeast rice, on the lipid pattern and on various cardiovascular end points in Chinese patients with a previous myocardial infarction. This study differs from all the other research projects because it is based on a very large number of patients with previous myocardial infarction (nearly 5000) who were treated for long time ( 4.5 years). Beside showing a significant increase of HDL-C as well as a significant decrease of TC, LDL-C, and TG, this study showed relevant clinical results. The frequency of nonfatal myocardial infarction and death from coronary heart disease showed an absolute and relative decrease of $4.7 \%$ and $45 \%$, respectively. Treatment with XZK also significantly decreased cardiovascular and total mortality by $30 \%$ and $33 \%$ and the need for coronary revascularization by $1 / 3$.

In conclusion, the effect of red yeast rice on HDL-C is still a matter of debate, because the only study that showed positive results follows a research protocol which differs from all the other studies. In this project, the selected population was constituted by patients with previous myocardial infarction. The strength of the study is that it has been conducted for a very long time and with recruitment of a very large population sample. The analysis of the data of Lu et al. shows that the effects of XZK on HDL-C may be caused at least in part by the potential properties of its nonstatin components. Thus, it is likely that components other than lovastatin in red yeast rice, such as lovastatin hydroxy acid, plant sterols, isoflavones, and isoflavone glycosides, may contribute to the results [28]. However, the future use of this product in clinical practice will depend on the separation, identification, characterization, and development of a carefully formulated preparation of red yeast rice as well as on additional studies that confirm its effect on a favorable lipid pattern in patients with dyslipidemia without previous myocardial infarction.

3.2. Olive Oil. Concerning the effects of olive oil on HDL$\mathrm{C}$, the literature search based on the keywords ["Olive Oil" OR "EVO" OR "Virgin Olive Oil”] AND ["HDL" OR "cholesterol" OR "high density lipoproteins"] retrieved 69 articles. After screening, 50 papers were selected for full-text revision. After applying our inclusion and exclusion criteria, 35 studies were excluded and 15 clinical trials were selected for the present systematic review. Figure 1 shows the study selection process. The 15 clinical trials studied a total of 1053 adults.

The trial characteristics are shown in Table 2. Regarding the study design, out of the 15 studies, two were randomized, double-blind, placebo-controlled, and parallel-groups trials $[32,42]$, two were randomized, double-blind, and controlled clinical trials $[30,35]$, one was a randomized and doubleblind trial [39], one was a randomized, controlled, and parallel study [44], one was a randomized, double-blind, and controlled crossover feeding trial [31], one was a randomized and single-blind crossover trial [33], two were randomized and controlled crossover studies [34,38], one was a randomized and intervention crossover trial [36], two were doubleblind crossover studies $[37,43]$, and two were pre-post studies $[40,41]$.

Regarding the control group, two studies used DHA $[30,32]$, one study used corn oil [31], two studies used low polyphenol olive oil $[34,36]$, one used virgin argan oil [44], and one used fish oil [30]. One study [33] had three control groups (Group A: EPA: 3 g/day; Group B: DHA: 3 g/day; Group C: fish oil: $3 \mathrm{~g} /$ day). One study [35] had two control groups (Group A: conjugated linoleic acid, free fatty acids: 27 g/day; Group B: conjugated linoleic acid, triacylglycerol: 27 g/day). Another study [37] had two control groups (Group A: $75 \mathrm{~g}$ /day of refined olive oil (OO); Group B: $75 \mathrm{~g} /$ day of common olive oil (a mixture of refined and virgin olive oil)).

One study had a control group which maintained its own dietary habits [39]. Four studies had no control group [38, 40, 41, 43].

The baseline characteristics and disease status of the participants varied. Four studies $[30,33,36,42]$ included subjects with hypercholesterolemia or moderate hypercholesterolemia; seven studies [37-41, 43, 44] included subjects with normal lipid levels, one study [32] included subject with adherence to a vegetarian diet for at least one year, and two studies $[34,35]$ included healthy males and healthy volunteers.

Dietary interventions lasted from 2 to 52 weeks with a dosage of extra virgin olive oil ranging from 2.28 to $54 \mathrm{~g} /$ day or a dosage of high polyphenol olive oil (HPOO) ranging from 22 to $69 \mathrm{~g} /$ day or with a dosage of virgin oil of $75 \mathrm{~g} /$ day. Two studies $[38,43]$ had three intervention groups (first 
study: Group A: HPOO (high polyphenol olive oil): 22 g/day; Group B: MPOO (medium polyphenol olive oil): $22 \mathrm{~g} /$ day; Group C: LPOO (low polyphenol olive oil): $22 \mathrm{~g} /$ day; second study: Group A: HPOO: 25 g/day; Group B: MPOO: 25 g/day; Group C: LPOO: 25 g/day).

Two studies $[32,38]$ showed a statistical significant change $(P<0.05)$ in HDL-C level between intervention and control groups. These three studies included a high number of participants; in particular, the study performed by Covas et al. in 2006 enrolled 200 subjects. Three studies [37, 39, $40]$ showed a significant difference $(\Delta$-changes $=0.08 ; 0.17$; $0.16 \mathrm{mmol} / \mathrm{L}, P<0.05)$ between pre- and posttreatment only in the intervention groups. These studies enrolled a small sample of participants ( $<100$ subjects). One study [44] showed a significant change in both the intervention group $(P<0.05)$ and the control group $(P<0.05)$. One crossover study [43] showed a significant difference in HDL-C levels in two out of the three intervention groups $(P<0.05$ in Group A and Group B). In the other three studies [30, 31, 36], no significant changes were observed, but it has to be considered that the selected population was characterized by normal or slightly increased TC values $(5,81,5.32$, and $4.19 \mathrm{mmol} / \mathrm{L}$ ) and with an HDL, respectively, equal to 1.21 , 1.09 , and $1.52 \mathrm{mmol} / \mathrm{L}$. In addition, these studies included a small sample of participants (57, 54, and 46 subjects).

In conclusion, these data provide evidence that polyphenol-rich olive oil favors the enhancement of HDL-C. The health-promoting properties of olive oil have traditionally been attributed to the oleic acid (omega-9 monounsaturated fatty acid, 18:1 n-9) component of olive oil. As a matter of fact, oleic acid enhances the levels of highdensity lipoprotein (HDL) and produces a small drop in low-density lipoprotein $[52,53]$. However, current knowledge indicates that olive oil polyphenols such as hydroxytyrosol play an important role in fortifying the healthy effects of olive oil [54]. As a phenolic acid, hydroxytyrosol decreases TC, LDL-C, and TG, while it increases HDL-C in hyperlipidemic rabbits and rats [55-58]. The purified polyphenols clinical studies are scarce and responsive mechanisms are not yet distinct. Olive oil polyphenols promote the main HDL antiatherogenic function, that is, its cholesterol efflux capacity. These polyphenols increase HDL size, promote a greater HDL stability reflected as a triglyceride-poor core, and enhance the HDL oxidative status, through an increase in the olive oil polyphenol metabolites content in the lipoprotein [34]. Covas et al. in 2006 with a 15-weeksupplementation of $2.2 \mathrm{~g}$ of the polyphenol content of olive oil showed further benefits on HDL-cholesterol levels and oxidative damage in addition to those obtained with its monounsaturated fatty acid content. This study provides additional evidence to recommend the use of polyphenolrich olive oil, that is, virgin olive oil, as a source of fat capable of promoting a reduction of some cardiovascular risk factors including the increase of HDL-C [38].

3.3. Bergamot. Concerning the effects of bergamot extract (BPF) on serum HDL-C, the literature search based on the keywords "bergamot" and ["HDL" OR "cholesterol" OR "high density lipoproteins"] retrieved 20 articles. After screening, 14 papers were selected for full-text revision. After applying our inclusion and exclusion criteria, 12 studies were excluded and 2 clinical trials were selected for the present systematic review. Figure 1 shows the study selection process. The 2 clinical trials studied a total of 314 adults (gender characteristics were not specified). The trial characteristics are outlined in Table 3 . Regarding the study design, one study was a randomized double-blind placebo-controlled trial [56], while the other one was an open label controlled trial [45].

Concerning the control group, Mollace et al's study [46] was placebo-controlled, whereas in Gliozzi et al.s study [45] there were 4 control groups (Group A: rosuvastatin $10 \mathrm{mg} /$ day; group B: rosuvastatin $20 \mathrm{mg}$ /day; group C: BPF $1000 \mathrm{mg}$ + rosuvastatin $10 \mathrm{mg}$ /day; and group D: placebo).

The baseline characteristics and disease status of the participants varied. Gliozzi et al's study [45] included subjects with mixed hypercholesterolemia, whereas in the other study 4 different groups were included: (i) subjects with hypercholesterolemia, (ii) hyperlipidemia (hypercholesterolemia and hypertriglyceridemia), (iii) hyperlipidemia and glycemia over $110 \mathrm{mg} / \mathrm{dL}$, and (iv) patients who discontinued statin therapy for muscular pain and a significant elevation of serum creatine phosphokinase (CPK). Dietary interventions lasted 4 weeks with a dosage of bergamot juice ranging from 500 to $1000 \mathrm{mg} / \mathrm{die}$.

One study [45] with a small sample of participants (77 subjects) showed a statistically significant increase $(P<0.05)$ of the HDL-C value. Similar results were obtained in the Mollace et al. study [46].

Similar results were obtained in study performed by Mollace et al. [46]. In this study a group of 59 patients diagnosed with metabolic syndrome were supplemented with BPF treatment with $500 \mathrm{mg} /$ day (group $\mathrm{Cl}, n=20$ ) or $1000 \mathrm{mg} /$ day (group C2, $n=19$ ) or placebo (group CPL, $n=20$ ). The metabolic syndrome patients, suffering from HC/HT/HG, responded very well to BPF therapy. After the treatment with high BPF dose, the patients with metabolic syndrome presented a significant mean increase $(+29.6 \%, P<$ 0.01 ) in HDL-C only in the $1000 \mathrm{mg}$ BPF treatment group (C2 group).

In conclusion, exogenous supply of bergamot extract which is rich in natural polyphenols favorably modulates various peripheral biomarkers of cardiometabolic risk, including the increase of HDL-cholesterol. In addition, a reduction of serum fasting glycaemia was observed. As a matter of fact, BPF inhibits HMG-CoA reductase activity and enhances reactive vasodilation, thus representing an efficient phytotherapeutic approach to fight glycemic and lipidemic disorders. A major contribution to the serum lipemic response observed in patients undergoing BPF treatment seems to be the modulatory properties of the flavonone glycoside components of the bergamot juice extract: in particular, naringin and neohesperidin seem to be of relevant interest.

Moreover, bergamot-derived polyphenols enhance the effect of rosuvastatin in normalizing the serum lipemic profile, allowing a potential reduction in daily rosuvastatin doses. The small number of studied patients as well as the availability of only two clinical studies coming from a unique research group suggests the need of additional research 
projects on this very interesting natural extract, in order to confirm the available data.

3.4. Artichoke. Concerning the effects of artichoke leaf extract (ALE) on HDL-C, the literature search based on the keywords ["Artichoke" OR "Artichoke Leaf Extract"] and ["HDL" OR "cholesterol" OR "high density lipoproteins"] retrieved 16 articles. After screening, 14 papers were selected for full-text revision. After applying our inclusion and exclusion criteria, 9 studies were excluded and 5 clinical trials were selected for the present systematic review. Figure 1 shows the study selection process. The 5 clinical trials studied a total of 395 adults.

The trial characteristics are outlined in Table 4. Regarding the study design, out of the 5 studies, three were randomized, double-blind, and placebo-controlled trials [47, $48,50]$, one was a randomized, double-blind, and placebocontrolled multicentre clinical trial [51], and one was a placebo-controlled trial [49]. The baseline characteristics and disease status of the participants varied. Two studies included subjects with hypercholesterolemia (Englisch et al. [51] TC: $7.7 \mathrm{mmol} / \mathrm{L}$; Bundy et al. [48] TC: $7.16 \mathrm{mmol} / \mathrm{L})$; one study included subjects with moderate hypercholesterolemia (mean value: $6.5 \mathrm{mmol} / \mathrm{L}$ ) [50]. The remaining two studies included subjects with type 2 diabetes without insulin therapy and with moderate hypercholesterolemia (mean values: 5.9 and $6.1 \mathrm{mmol} / \mathrm{L}$, resp.). In one of these two studies, the mean basal HDL-C value was low $(0.88 \mathrm{mmol} / \mathrm{L})$ [49], while it was high in the other one $(1.46 \mathrm{mmol} / \mathrm{L})$ [47].

Dietary interventions lasted from 6 to 12 weeks (mean value: 64 days) with a supplementation of artichoke leaf extract (dosage from $0.5 \mathrm{~g} /$ day to $6 \mathrm{~g} /$ day), dry extract (dosage of $1.8 \mathrm{~g} /$ day), and flowering bud leaf extract (dosage of $0.6 \mathrm{~g} /$ day).

One study [50] showed a statistically significant difference $(\Delta$-changes $=+0.20 \mathrm{mmol} / \mathrm{L}, P<0.01,95 \% \mathrm{CI}=0.07 ; 0.34)$ in the level of HDL-C when intervention and control group were compared. Similar results were obtained in Nazni et al. study [49] when pre-post intervention data were compared $(\Delta$ change $=+0.22 \mathrm{mmol} / \mathrm{L})$. In the other three studies $[47,48$, 51], no significant HDL-C changes were observed. Anyhow, looking carefully at the mean basal lipemic values of the selected samples of all the five studies, a significant increase of HDL-C was not obtained when the mean TC value was higher than 7.16 or when mean HDL-C was higher than $1.46 \mathrm{mmol} / \mathrm{L}$ and the three negative studies showed basal lipemic values above these values.

In conclusion, ALE supplementation appears a useful treatment to increase HDL-C provided that basal TC or basal HDL-C is not too high. Therefore, ALE could represent a potential treatment option for subjects with primary mild hypercholesterolaemia, particularly if HDL values are low. ALE also proved to be safe and well tolerated. Future studies are needed to confirm the favorable clinical results and safety over a longer duration of intervention with ALE.

Although the mechanisms explaining the HDLcholesterol increasing effect of ALE are not well known, the most likely explanation could be related to their polyphenolic content and in particular to chlorogenic acid [59-61]. This compound could favor the increase in HDL-C through the enhancement of the activity of paraoxonase1 (PON-1). PON-1 is an enzyme associated with HDL which prevents the oxidation of HDL-C, thus favoring its antioxidative and anti-inflammatory effects [62]. Gugliucci and Bastos demonstrated that chlorogenic acid protects PON-1 activity in HDL-C by means of an in vitro study. In this experiment, chlorogenic acid protects PON-1 activity in human HDLs from inactivation caused by hypochlorite $(\mathrm{HOCl})$, at concentrations of $\mathrm{HOCl}(50 \mu \mathrm{M})$ and chlorogenic acid $(2-10 \mu \mathrm{M})$ compatible with those found in humans [63].

Previous human studies proved that the increase in PON1 activity correlates strongly with the increase in HDL-C $[64,65]$. Based on these data, it can be hypothesized that chlorogenic acid, present in ALE extract, may induce an increase in PON-1 activity and, as a consequence, may favor the increase in HDL-C in humans.

\section{Conclusions}

The results of this systematic review indicate that the dietary supplementation with four typical components of the MediterrAsian diet, that is, red yeast rice, bergamot, artichoke, and virgin olive oil, has promising effects on the increase of HDL-cholesterol serum levels. In Figure 2, variation of HDL-C in mmol after intervention has been shown. The artichoke leaf extract and virgin olive oil appear to be particularly interesting: for both these products, a good number of papers are available as well as a large number of studied subjects. Moreover, the entity of the HDL-C increase appears to be relevant after supplementation with both the artichoke leaf extract and the virgin olive oil, while this result does not appear with "nonvirgin" olive oil. Anyhow, a new meta-analysis of the existing data should be planned for both of them, in order to obtain a more accurate and evidence-based information. Additional research should be planned on bergamot extract because the available results on this botanical appear very interesting but based on a too small number of studies (two papers coming from a unique research group) and of evaluated subjects. The effect of red yeast rice on HDL-C appears positive only after long term intervention in patients with previous myocardial infarction; while negative effects are shown in multiple studies conducted in asymptomatic dyslipidemic subjects. This finding could play a role in the treatment of patients with positive history of myocardial infarction, but this finding needs further confirmation by means of additional controlled intervention studies. The effects of these MediterrAsian natural extracts and food products on HDL-C changes appear very promising, but further research by means of additional well conducted, randomized, controlled studies in a large male and female population with low HDL-C values is needed. Moreover, the effect on both HDL-cholesterol and HDL particles should be evaluated, as well as the correlation between the increase in HDL-cholesterol and nonfunctional HDL particles. 


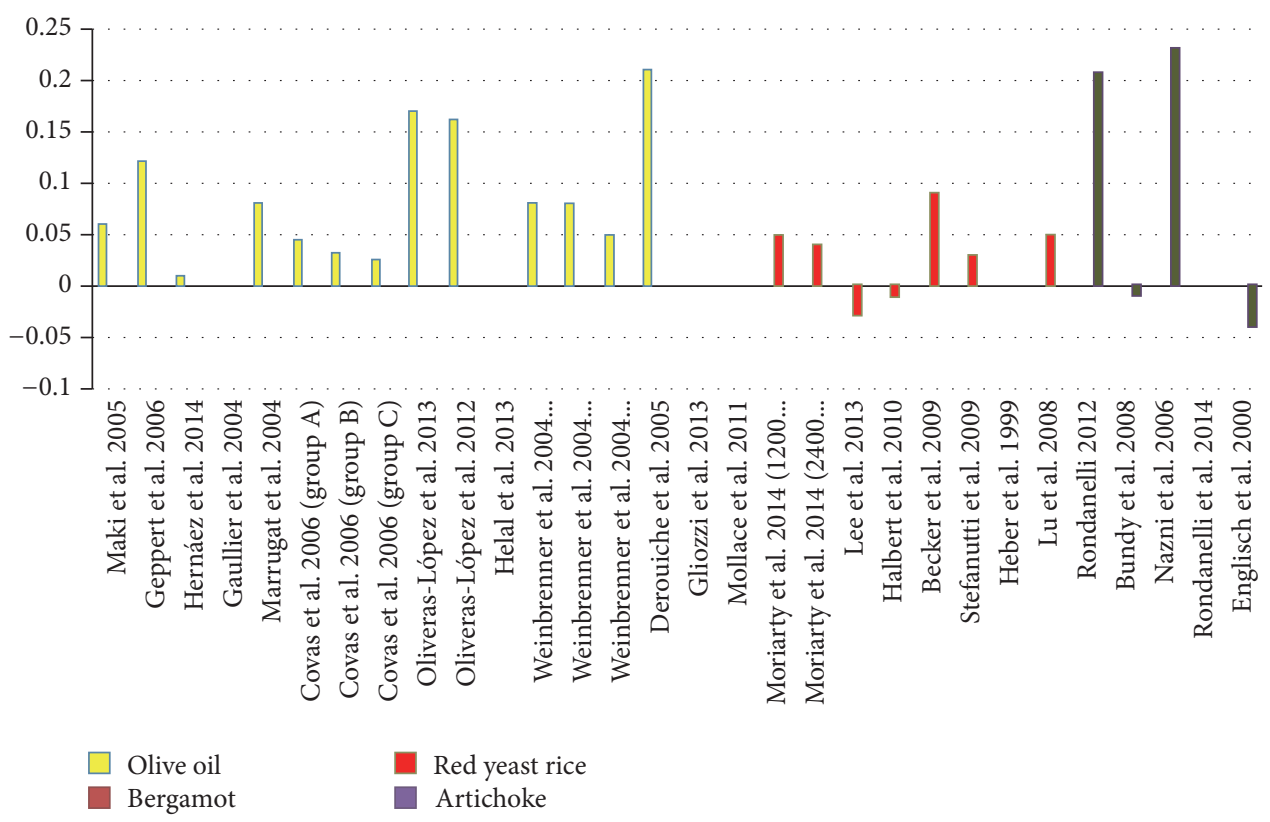

FIGURE 2: Variation of HDL-C in mmol after intervention.

\section{Competing Interests}

The authors declare no conflict of interests regarding the publication of this paper.

\section{References}

[1] C. J. L. Murray and A. D. Lopez, "Global mortality, disability, and the contribution of risk factors: global burden of disease study," The Lancet, vol. 349, no. 9063, pp. 1436-1442, 1997.

[2] K. S. Jain, M. K. Kathiravan, R. S. Somani, and C. J. Shishoo, "The biology and chemistry of hyperlipidemia," Bioorganic and Medicinal Chemistry, vol. 15, no. 14, pp. 4674-4699, 2007.

[3] G. De Backer, E. Ambrosioni, K. Borch-Johnsen et al., "European guidelines on cardiovascular disease prevention in clinical practice: third joint task force of European and other societies on cardiovascular disease prevention in clinical practice (constituted by representatives of eight societies and by invited experts)," European Journal of Cardiovascular Prevention and Rehabilitation, vol. 10, no. 4, pp. S1-S10, 2003.

[4] D. J. Gordon, J. L. Probstfield, R. J. Garrison et al., "Highdensity lipoprotein cholesterol and cardiovascular disease. Four prospective American studies," Circulation, vol. 79, no. 1, pp. 8$15,1989$.

[5] A. M. Gotto, S. J. Boccuzzi, J. R. Cook et al., "Effect of lovastatin on cardiovascular resource utilization and costs in the Air Force/Texas Coronary Atherosclerosis Prevention Study (AFCAPS/TexCAPS). AFCAPS/TexCAPS Research Group," American Journal of Cardiology, vol. 86, pp. 1176-1181, 2000.

[6] J. Shepherd, S. M. Cobbe, I. Ford et al., "Prevention of coronary heart disease with pravastatin in men with hypercholesterolemia," The New England Journal of Medicine, vol. 333, no. 20, pp. 1301-1307, 1995.

[7] F. M. Sacks, M. A. Pfeffer, L. A. Moye et al., "The effect of pravastatin on coronary events after myocardial infarction in patients with average cholesterol levels. Cholesterol and
Recurrent Events Trial investigators," The New England Journal of Medicine, vol. 335, no. 14, pp. 1001-1009, 1996.

[8] Scandinavian Simvastatin Survival Study Group, "Randomised trial of cholesterol lowering in 4444 patients with coronary heart disease: the Scandinavian Simvastatin Survival Study (4S)," The Lancet, vol. 344, no. 8934, pp. 1383-1389, 1994.

[9] M. J. Chapman, "Are the effects of statins on HDL-cholesterol clinically relevant?" European Heart Journal Supplements, vol. 6, pp. C58-C63, 2004.

[10] R. B. Kelly, "Diet and exercise in the management of hyperlipidemia," American Family Physician, vol. 81, no. 9, pp. 1097-1102, 2010.

[11] W. C. Willett, F. Sacks, A. Trichopoulou et al., "Mediterranean diet pyramid: a cultural model for healthy eating," The American Journal of Clinical Nutrition, vol. 61, no. 6, supplement, pp. 1402S-1406S, 1995.

[12] T. A. Nicklas, C. E. O'Neil, and V. L. Fulgoni, "Rice consumption is associated with better nutrient intake and diet quality in adults: National Health and Nutrition Examination survey (NHANES) 2005-2010," Food and Nutrition Sciences, vol. 5, pp. 525-532, 2014.

[13] K. Pallauf, K. Giller, P. Huebbe, and G. Rimbach, "Nutrition and healthy ageing: calorie restriction or polyphenolrich 'MediterrAsian' diet?" Oxidative Medicine and Cellular Longevity, vol. 2013, Article ID 707421, 14 pages, 2013.

[14] E. A. Brinton, "Effects of ethanol intake on lipoproteins," Current Atherosclerosis Reports, vol. 14, no. 2, pp. 108-114, 2012.

[15] J. M. Miranda, X. Anton, C. Redondo-Valbuena et al., "Egg and egg-derived foods: effects on human health and use as functional foods," Nutrients, vol. 7, no. 1, pp. 706-729, 2015.

[16] C. J. Andersen, C. N. Blesso, J. Lee et al., "Egg consumption modulates HDL lipid composition and increases the cholesterol-accepting capacity of serum in metabolic syndrome," Lipids, vol. 48, no. 6, pp. 557-567, 2013.

[17] M. Egger, K. Dickersin, and G. D. Smith, "Problems and limitations in conducting systematic reviews," in Systematic 
Reviews in Health Care: Meta-Analysis in Context, M. Egger, G. D. Smith, and D. G. Altman, Eds., BMJ Books, London, UK, 2nd edition, 2001.

[18] National Library of Medicine, Bethesda, Md, USA, http://www pubmed.com.

[19] http://www.scopus.com/.

[20] http://thomsonreuters.com/en.html.

[21] http://www.scholar.google.com.

[22] P. M. Moriarty, E. M. Roth, A. Karns et al., "Effects of Xuezhikang in patients with dyslipidemia: a multicenter, randomized, placebo-controlled study," Journal of Clinical Lipidology, vol. 8, no. 6, pp. 568-575, 2014.

[23] C.-Y. Lee, M.-S. Jan, M.-C. Yu, C.-C. Lin, J. C.-C. Wei, and H.-C. Shih, "Relationship between adiponectin and leptin, and blood lipids in hyperlipidemia patients treated with red yeast rice," Forschende Komplementärmedizin, vol. 20, no. 3, pp. 197-203, 2013.

[24] S. C. Halbert, B. French, R. Y. Gordon et al., "Tolerability of red yeast rice (2,400 $\mathrm{mg}$ twice daily) versus Pravastatin $(20 \mathrm{mg}$ twice daily) in patients with previous statin intolerance," American Journal of Cardiology, vol. 105, no. 2, pp. 198-204, 2010.

[25] D. J. Becker, R. Y. Gordon, S. C. Halbert, B. French, P. B. Morris, and D. J. Rader, "Red yeast rice for dyslipidemia in statin-intolerant patients: a randomized trial," Annals of Internal Medicine, vol. 150, no. 12, pp. 830-839, 2009.

[26] C. Stefanutti, F. Mazza, A. Vivenzio et al., "Combined treatment with Dif1stat ${ }^{\circledR}$ and diet reduce plasma lipid indicators of moderate hypercholesterolemia more effectively than diet alone: a randomized trial in parallel groups," Lipids, vol. 44, no. 12, pp. 1141-1148, 2009.

[27] D. Heber, I. Yip, J. M. Ashley, D. A. Elashoff, R. M. Elashoff, and V. L. W. Go, "Cholesterol-lowering effects of a proprietary Chinese red-yeast-rice dietary supplement," American Journal of Clinical Nutrition, vol. 69, no. 2, pp. 231-236, 1999.

[28] Z. Lu, W. Kou, B. Du et al., "Effect of xuezhikang, an extract from red yeast chinese rice, on coronary events in a chinese population with previous myocardial infarction," American Journal of Cardiology, vol. 101, no. 12, pp. 1689-1693, 2008.

[29] Centre for Evidence-Based Medicine. Levels of Evidence. New Levels of Evidence Table, http://www.cebm.net/index .aspx?o=5653.

[30] K. C. Maki, A. L. Lawless, K. M. Kelley, V. N. Kaden, C. J. Geiger, and M. R. Dicklin, "Corn oil improves the plasma lipoprotein lipid profile compared with extra-virgin olive oil consumption in men and women with elevated cholesterol: results from a randomized controlled feeding trial," Journal of Clinical Lipidology, vol. 9, no. 1, pp. 49-57, 2015.

[31] K. C. Maki, M. E. Van Elswyk, D. McCarthy et al., "Lipid responses to a dietary docosahexaenoic acid supplement in men and women with below average levels of high density lipoprotein cholesterol," Journal of the American College of Nutrition, vol. 24, no. 3, pp. 189-199, 2005.

[32] J. Geppert, V. Kraft, H. Demmelmair, and B. Koletzko, "Microalgal docosahexaenoic acid decreases plasma triacylglycerol in normolipidaemic vegetarians: a randomised trial," British Journal of Nutrition, vol. 95, no. 4, pp. 779-786, 2006.

[33] G. S. Rambjør, A. I. Wålen, S. L. Windsor, and W. S. Harris, "Eicosapentaenoic acid is primarily responsible for hypotriglyceridemic effect of fish oil in humans," Lipids, vol. 31, no. 1, pp. S45-S49, 1996.
[34] Á. Hernáez, S. Fernández-Castillejo, M. Farràs et al., "Olive oil polyphenols enhance high-density lipoprotein function in humans: a randomized controlled trial," Arteriosclerosis, Thrombosis, and Vascular Biology, vol. 34, no. 9, pp. 2115-2119, 2014.

[35] J. M. Gaullier, J. Halse, K. Høye et al., "Conjugated linoleic acid supplementation for $1 \mathrm{y}$ reduces body fat mass in healthy overweight humans," The American Journal of Clinical Nutrition, vol. 79, no. 6, pp. 1118-1125, 2004.

[36] M. N. Vissers, P. L. Zock, S. A. Wiseman, S. Meyboom, and M. B. Katan, "Original communications-effect of phenol-rich extra virgin olive oil on markers of oxidation in healthy volunteers," European Journal of Clinical Nutrition, vol. 55, pp. 334-341, 2001.

[37] J. Marrugat, M. I. Covas, M. Fitó et al., "Effects of differing phenolic content in dietary olive oils on lipids and LDL oxidation," European Journal of Nutrition, vol. 43, no. 3, pp. 140147, 2004.

[38] M.-I. Covas, K. Nyyssönen, H. E. Poulsen et al., "The effect of polyphenols in olive oil on heart disease risk factors: A randomized trial," Annals of Internal Medicine, vol. 145, no. 5, pp. 333-341, 2006.

[39] M.-J. Oliveras-López, J. J. M. Molina, M. V. Mir, E. F. Rey, F. Martín, and H. L.-G. de la Serrana, "Extra virgin olive oil (EVOO) consumption and antioxidant status in healthy institutionalized elderly humans," Archives of Gerontology and Geriatrics, vol. 57, no. 2, pp. 234-242, 2013.

[40] M.-J. Oliveras-López, M. Innocenti, F. Martín Bermudo, H. López-García de la Serrana, and N. Mulinacci, "Effect of extra virgin olive oil on glycaemia in healthy young subjects," European Journal of Lipid Science and Technology, vol. 114, no. 9, pp. 999-1006, 2012.

[41] O. Helal, H. Berrougui, S. Loued, and A. Khalil, "Extra-virgin olive oil consumption improves the capacity of HDL to mediate cholesterol efflux and increases ABCA1 and ABCG1 expression in human macrophages," British Journal of Nutrition, vol. 109, no. 10, pp. 1844-1855, 2013.

[42] J. A. Lovegrove, S. S. Lovegrove, S. V. Lesauvage et al., "Moderate fish-oil supplementation reverses low-platelet, long-chain n-3 polyunsaturated fatty acid status and reduces plasma triacylglycerol concentrations in British Indo-Asians," The American Journal of Clinical Nutrition, vol. 79, no. 6, pp. 974-982, 2004.

[43] T. Weinbrenner, M. Fitó, R. De La Torre et al., "Olive oils high in phenolic compounds modulate oxidative/antioxidative status in men," Journal of Nutrition, vol. 134, no. 9, pp. 2314-2321, 2004.

[44] A. Derouiche, M. Cherki, A. Drissi et al., "Nutritional intervention study with argan oil in man: effects on lipids and apolipoproteins," Annals of Nutrition and Metabolism, vol. 49, no. 3, pp. 196-201, 2005.

[45] M. Gliozzi, R. Walker, S. Muscoli et al., "Bergamot polyphenolic fraction enhances rosuvastatin-induced effect on LDLcholesterol, LOX-1 expression and protein kinase B phosphorylation in patients with hyperlipidemia," International Journal of Cardiology, vol. 170, no. 2, pp. 140-145, 2013.

[46] V. Mollace, I. Sacco, E. Janda et al., "Hypolipemic and hypoglycaemic activity of bergamot polyphenols: from animal models to human studies," Fitoterapia, vol. 82, no. 3, pp. 309-316, 2011.

[47] M. Rondanelli, A. Opizzi, M. Faliva et al., "Metabolic management in overweight subjects with naive impaired fasting glycaemia by means of a highly standardized extract from cynara scolymus: a double-blind, placebo-controlled, randomized clinical trial," Phytotherapy Research, vol. 28, no. 1, pp. 3341, 2014. 
[48] R. Bundy, A. F. Walker, R. W. Middleton, C. Wallis, and H. C. R. Simpson, "Artichoke leaf extract (Cynara scolymus) reduces plasma cholesterol in otherwise healthy hypercholesterolemic adults: a randomized, double blind placebo controlled trial," Phytomedicine, vol. 15, no. 9, pp. 668-675, 2008.

[49] P. Nazni, T. Poongodi Vijayakumar, P. Alagianambi, and M. Amirthaveni, "Hypoglycemic and hypolipidemic effect of Cynara scolymus among selected type 2 diabetic individuals," Pakistan Journal of Nutrition, vol. 5, no. 2, pp. 147-151, 2006.

[50] M. Rondanelli, A. Giacosa, A. Opizzi et al., "Beneficial effects of artichoke leaf extract supplementation on increasing HDLcholesterol in subjects with primary mild hypercholesterolaemia: a double-blind, randomized, placebo-controlled trial," International Journal of Food Sciences and Nutrition, vol. 64, no. 1, pp. 7-15, 2013.

[51] W. Englisch, C. Beckers, M. Unkauf, M. Ruepp, and V. Zinserling, "Efficacy of artichoke dry extract in patients with hyperlipoproteinemia," Arzneimittel-Forschung/Drug Research, vol. 50, no. 3, pp. 260-265, 2000.

[52] M. B. Katan, P. L. Zock, and R. P. Mensink, "Effects of fats and fatty acids on blood lipids in humans: an overview," American Journal of Clinical Nutrition, vol. 60, supplement 6, pp. 1017S1022S, 1994.

[53] M. B. Katan, P. L. Zock, and R. P. Mensink, "Dietary oils, serum lipoproteins, and coronary heart disease," The American Journal of Clinical Nutrition, vol. 61, no. 6, supplement, pp. 1368S-1373S, 1995.

[54] S. Granados-Principal, J. L. Quiles, C. L. Ramirez-Tortosa, P. Sanchez-Rovira, and M. C. Ramirez-Tortosa, "Hydroxytyrosol: from laboratory investigations to future clinical trials," Nutrition Reviews, vol. 68, no. 4, pp. 191-206, 2010.

[55] M. González-Santiago, E. Martín-Bautista, J. J. Carrero et al., "One-month administration of hydroxytyrosol, a phenolic antioxidant present in olive oil, to hyperlipemic rabbits improves blood lipid profile, antioxidant status and reduces atherosclerosis development," Atherosclerosis, vol. 188, no. 1, pp. 35-42, 2006.

[56] I. Fki, Z. Sahnoun, and S. Sayadi, "Hypocholesterolemic effects of phenolic extracts and purified hydroxytyrosol recovered from olive mill wastewater in rats fed a cholesterol-rich diet," Journal of Agricultural and Food Chemistry, vol. 55, no. 3, pp. 624-631, 2007.

[57] H. Jemai, I. Fki, M. Bouaziz et al., "Lipid-lowering and antioxidant effects of hydroxytyrosol and its triacetylated derivative recovered from olive tree leaves in cholesterol-fed rats," Journal of Agricultural and Food Chemistry, vol. 56, no. 8, pp. 26302636, 2008.

[58] H. Jemai, M. Bouaziz, and S. Sayadi, "Phenolic composition, sugar contents and antioxidant activity of Tunisian sweet olive cultivar with regard to fruit ripening," Journal of Agricultural and Food Chemistry, vol. 57, no. 7, pp. 2961-2968, 2009.

[59] J. E. Brown and C. A. Rice-Evans, "Luteolin-rich artichoke extract protects low density lipoprotein from oxidation in vitro," Free Radical Research, vol. 29, no. 3, pp. 247-255, 1998.

[60] R. Gebhardt, "Inhibition of cholesterol biosynthesis in HepG2 cells by artichoke extracts is reinforced by glucosidase pretreatment," Phytotherapy Research, vol. 16, no. 4, pp. 368-372, 2002.

[61] B. Wider, M. H. Pittler, J. Thompson-Coon, and E. Ernst, "Artichoke leaf extract for treating hypercholesterolaemia," Cochrane Database of Systematic Reviews, no. 4, Article ID CD003335, 2009.
[62] C. Gouédard, R. Barouki, and Y. Morel, "Dietary polyphenols increase paraoxonase 1 gene expression by an aryl hydrocarbon receptor-dependent mechanism," Molecular and Cellular Biology, vol. 24, no. 12, pp. 5209-5222, 2004.

[63] A. Gugliucci and D. H. M. Bastos, "Chlorogenic acid protects paraoxonase 1 activity in high density lipoprotein from inactivation caused by physiological concentrations of hypochlorite," Fitoterapia, vol. 80, no. 2, pp. 138-142, 2009.

[64] M. S. van der Gaag, A. van Tol, L. M. Scheek et al., "Daily moderate alcohol consumption increases serum paraoxonase activity; a diet-controlled, randomised intervention study in middle-aged men," Atherosclerosis, vol. 147, no. 2, pp. 405-410, 1999.

[65] A. Sierksma, M. S. van der Gaag, A. van Tol, R. W. James, and H. F. J. Hendriks, "Kinetics of HDL cholesterol and paraoxonase activity in moderate alcohol consumers," Alcoholism: Clinical and Experimental Research, vol. 26, no. 9, pp. 1430-1435, 2002. 

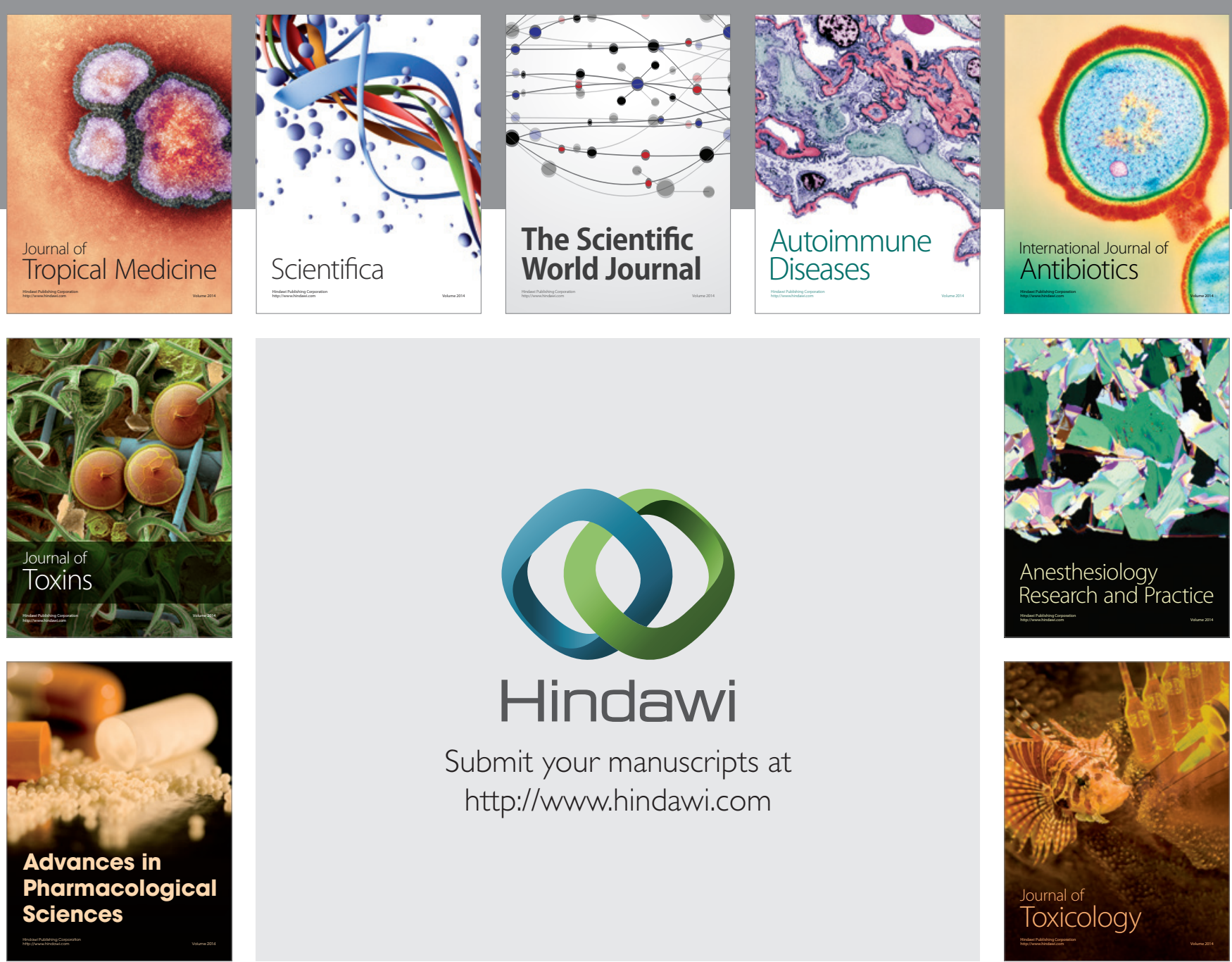

\section{Hindawi}

Submit your manuscripts at

http://www.hindawi.com
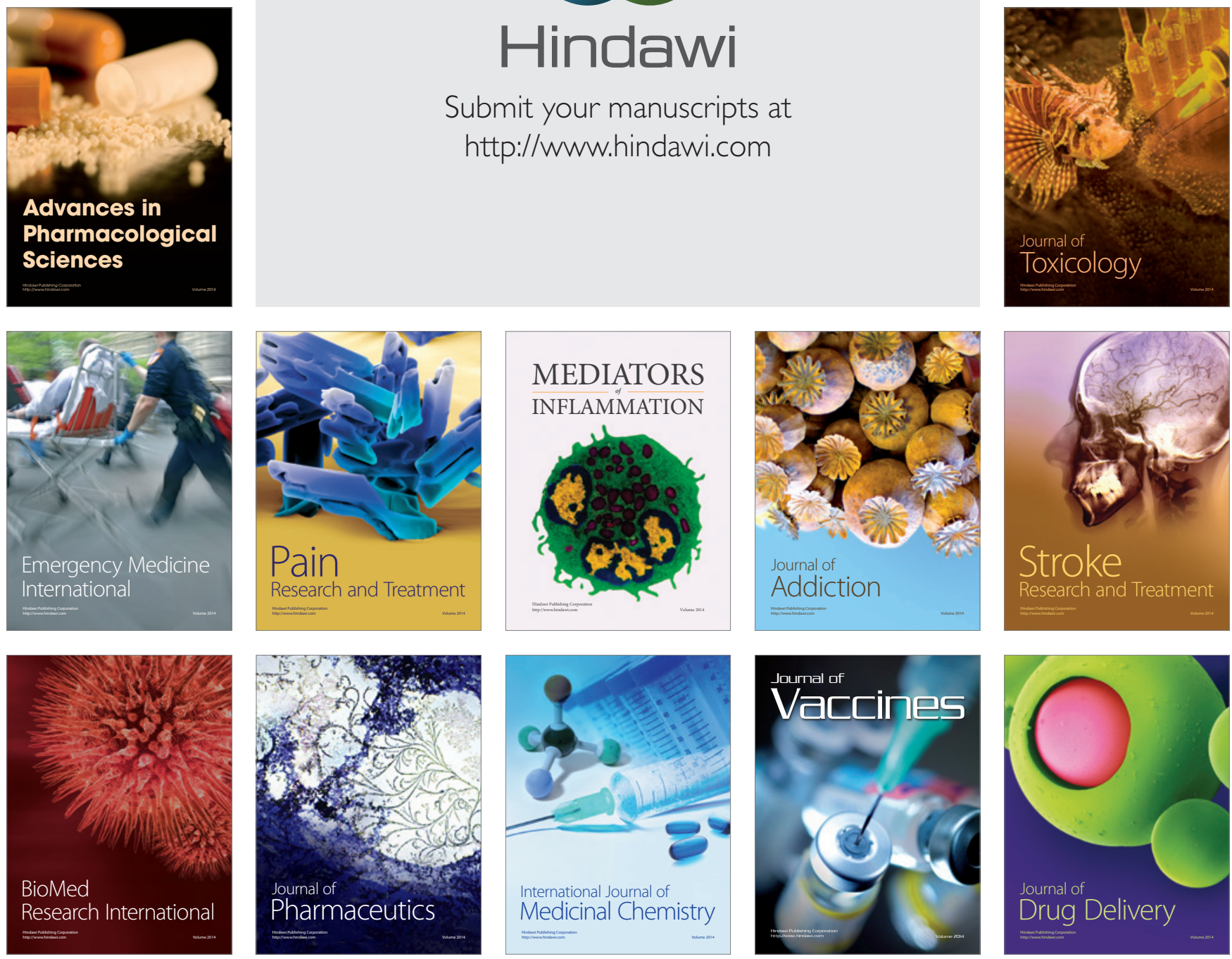